\title{
Two types of coronal bright points their characteristics, and evolution
}

\author{
Isroil Sattarov ${ }^{1}$, Nina V. Karachik ${ }^{2}$, Chori T. Sherdanov ${ }^{3}$, \\ Azlarxon M. Tillaboev ${ }^{1}$, and Alexei A. Pevtsov ${ }^{2}$ \\ ${ }^{1}$ Tashkent State Pedagogical University, 103 Yusif Kxos Kxojib str., \\ Tashkent 100070, Uzbekistan \\ email: isattar@astrin.uz \\ ${ }^{2}$ National Solar Observatory, Sunspot, NM 88349, USA \\ email: apevtsov@nso.edu \\ ${ }^{3}$ Astronomical Institute of the Academy of Sciences of Uzbekistan \\ email: chori@astrin.uz
}

\begin{abstract}
Using maximum brightness of coronal bright point's (CBP) as a criterion, we separate them on two categories: dim CBPs, associated with areas of a quiet Sun, and bright CBPs, associated with an active Sun. This study reports on characteristics of two types of CBPs and their evolution.
\end{abstract}

Keywords. Solar corona, coronal bright points

\section{Introduction and Data}

Coronal Bright Points (CBPs, also referred to as X-ray bright points, XBPs) are compact coronal brightenings observed in X-ray, EUV, and radiowaves. Several authors had reported on existence of two types of XBPs: XBPs of quiet Sun, uniformly distributed over the solar surface (both temporally and spatially) and the XBPs with non-statistical variations in the longitudinal distributions e.g. Golub, Krieger, Vaiana (1975); Sattarov, et al. (2002, 2005a,b); McIntosh \& Gurman (2005). Sattarov (2007) have found that the number of high latitude CBPs shows negative correlation with sunspot cycle, whereas number of CBPs in active region belts correlates positively with the cycle. This finding suggests a more complicated relationship between the CBP number and the solar activity cycle than had been previously thought. In this report we separate CBPs on two types using their brightness: "dim" CBPs are connected to quiet Sun regions and "bright" CBPs related to the active Sun. We investigate the characteristics of the two types of CBPs and their evolution, including the latitudinal distribution, temporal variation of CBP's maximal intensity, area, and the lifetime. We use full disk images observed by the Extreme-ultraviolet Imaging Telescope (EIT) on board of SOHO. We utilize EIT full disk synoptic data with spatial resolution of 2.64 arcsec per pixel and six hours cadence observed (full disk mode) in $195 \AA$ from 1996-2008. For study the evolution of CBPs, we use EIT data obtained in "CME watch" mode with much higher time cadence. The data are calibrated following the standard EIT data reduction routine. To identify the coronal bright points, we employ the automatic procedure developed by us see (Sattarov, et al. 2010).

\section{Temporal and latitudinal distribution of CBPs}

If the CBPs are related to magnetic fields in the photosphere, one can make a reasonable assumption that the intensity of CBP must correlate with the magnetic flux. As is 


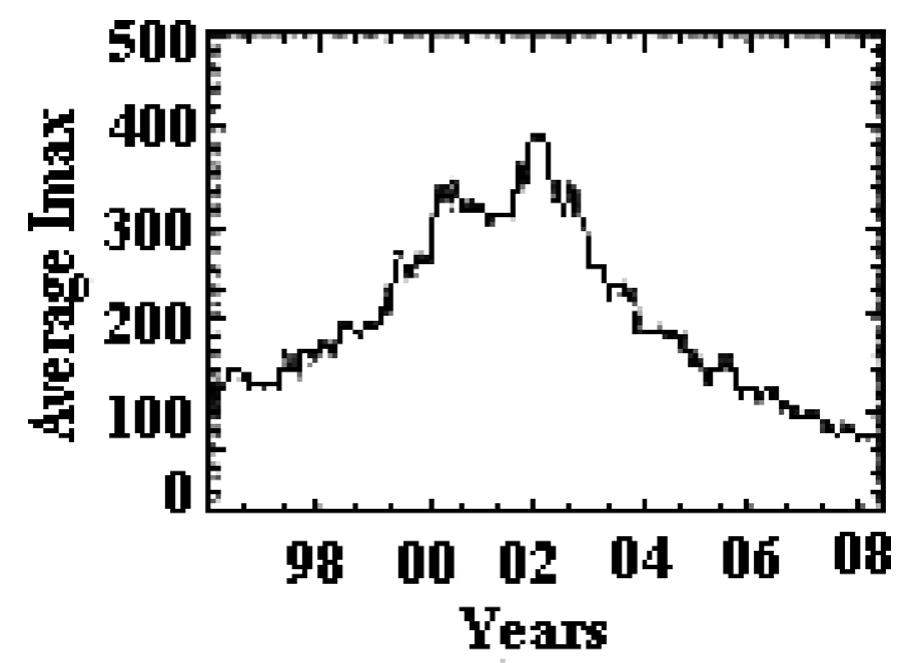

Figure 1. Cycle variation of average maximal intensity (Imax) of CBP for 1996-2008.

well-known, the magnetic flux on solar surface changes with solar activity, and therefore the increased level of solar activity would stimulate rise of maximal intensity of CBP and its decrease would followed by a decline of CBP brightness. Figure 1 presents cycle variation of average maximal intensity of CPBs during solar Cycle 23, which indicates that CBPs are (on average) brighter during maximum of solar activity cycle as compared with periods of solar minimum. At solar minimum in 1996, the average Imax intensity was about 150 DNs. Thus, we take it as a threshold between the "dim" and "bright" CBPs. In accordance with this criteria at solar minimum (1996) a majority of CBPs is "dim" ones. In 1997, number of bright CBPs starts rise quickly, while number of "dim" CBPs decreases. Latitudinal distribution of "dim" CBPs for 1996 (see Fig.6 in Sattarov, et al. (2010)) follows cosine function . The latitudinal distribution of bright CBPs (See Fig. 7 in Sattarov, et al. (2010)) for 1998 and 2002 exhibits two humps similar to latitudinal distribution of sunspots. However, in these years of high solar activity, both types of CBPs are present; bright CBP being predominant in active region belts, and dim CBPs are more abundant near the equator.

\section{Evolution of coronal bright points}

To study temporal evolution of CBPs we use EIT data obtained in "CME Watch" mode on 26 September 1997 (start of cycle 23) and 21 December 2002 (second maximum of the cycle). We find that CBPs have different area at maximum of its brightness, from 10 up to 200 pixels, and we study evolution of CBPs of different area (Figure 2).

On Fig.2 one can see the evolutions of two types CBP have small difference by area, but amplitude variation of intensity differs. The difference is small for bright CBP and large for "dim" ones. Fig. 3 presents the temporal variations in intensity and area of small CBP of quiet Sun type. A small flare-like brightening had occurred at the beginning of CBP development (see peak in intensity in Figure 3, right). After the intensity spike, CBP area remains glaring during 5-10 hour. CBPs of larger size also show flare-like changes in intensity (see examples in Figure 2). Area of CBP shown in Figure 2 is 8 pixels or circle with radius 4 arc sec or 16 MHS. It represents an example of small, quiet Sun CBP during solar minimum of activity. Based on the examples shown in Figure 2, we speculate that 

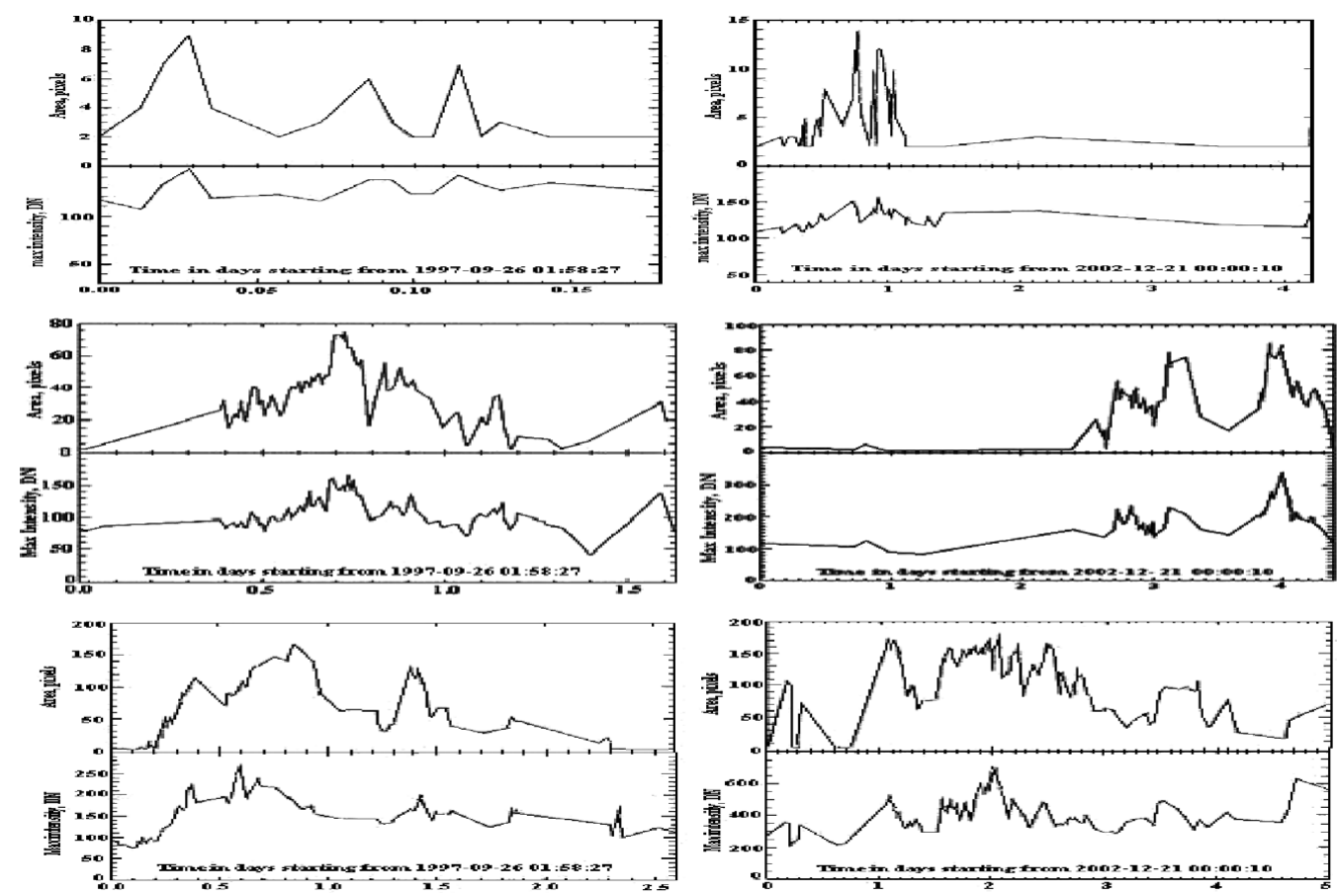

Figure 2. Examples of temporal variation an area (upper panel for each example) and intensity (lower panel for each example) of CBPs of different size and maximum intensity for periods of low (left column) and high (right) levels of solar activity.
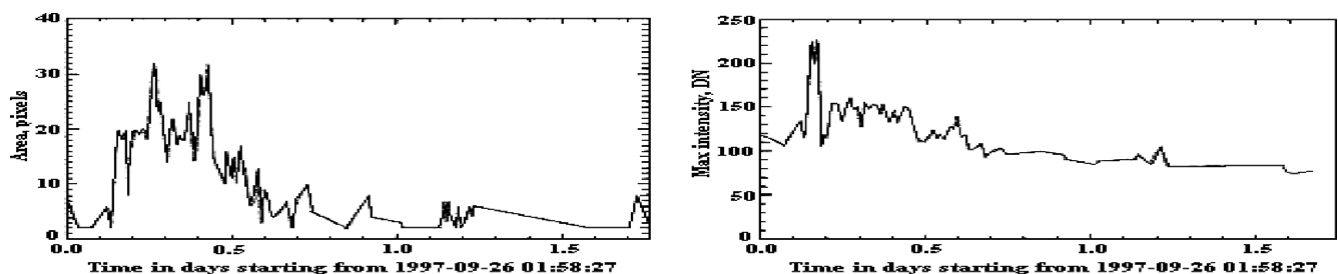

Figure 3. Temporal variation an area and intensity for CBP on September 261997.

large CBPs may be comprised of smaller CBPs that exhibit flare-like activity similar to example shown in Figure 3.

\section{Discussion and Conclusions}

We find that the active and quiet Sun coronal bright points exhibit flare-like variation in their maximum intensity. The amplitude of intensity variations is higher for small CBPs and lower for larger ones. Small quiet Sun CBPs may show repeated flare-like spikes with amplitude gradually decreasing during half a day period. This flaring slowly increases background around the CBP. Large CBPs also show such repeated flaring up albeit with smaller amplitude as compared with small CBPs. The repeat flaring in large CBPs may be related to elementary CBPs that collectively form large CBP. This our finding is in agreement with early studies, which found that X-ray bright point may consist of several kernels that flare-up one after the other. Small CBPs may consist of a 
single kernel (one elementary CBP), which would explain higher amplitude variations in their intensity as compared with larger CBPs.

\section{Acknowledgements}

The presentation of this paper at the IAU Symposium 273 was made possible due to partial support from the National Science Foundation grant numbers ATM 0548260, AST 0968672 and NASA - Living With a Star grant number 09-LWSTRT09-0039.

\section{References}

Golub, L., Krieger, A. S., \& Vaiana, G. S. 1975, Solar Phys., 42, 131

McIntosh, S. W. \& Gurman, J. B. 2005, Solar Phys., 228, 285

Sattarov, I., Pevtsov, A. A., Karachik, N. V., Sherdanov, C. T., \& Tillaboev, A. M. 2010, Solar Phys., 262, 321

Sattarov, I., Pevtsov, A. A., Karachik, N. V., \& Sherdanov, C. T. 2007, Adv. Sp. Res. 39, 1777

Sattarov, I., Pevtsov, A. A., Karachik, N. V., \& Sattarova, B. J. 2005a, ASP Conf. Ser., 346, 363

Sattarov, I., Pevtsov, A. A., Karachik, N. V., \& Sattarova, B. J. 2005b, ASP Conf. Ser., 346, 395

Sattarov, I., Pevtsov, A. A., Hojaev, A. S., \& Sherdonov, C. T. 2002, Astrophys. J., 564, 1042 